\title{
Radiofrequency ablation for hepatocellular carcinoma induces glypican-3 peptide-specific cytotoxic T lymphocytes
}

\author{
DAISUKE NOBUOKA ${ }^{1,4^{*}}$, YUTAKA MOTOMURA ${ }^{1,5^{*}}$, HIROFUMI SHIRAKAWA $^{1}$, \\ TOSHIAKI YOSHIKAWA ${ }^{1}$, TOSHIMITSU KURONUMA ${ }^{1}$, MARI TAKAHASHI ${ }^{1}$, KOHEI NAKACHI ${ }^{2}$, \\ HIROSHI ISHII ${ }^{2}$, JUNJI FURUSE ${ }^{2}$, NAOTO GOTOHDA ${ }^{3}$, SHINICHIRO TAKAHASHI ${ }^{3}$, \\ TOSHIO NAKAGOHRI ${ }^{3}$, MASARU KONISHI $^{3}$, TAIRA KINOSHITA ${ }^{3}$, HIROYUKI KOMORI $^{5}$, \\ HIDEO BABA ${ }^{5}$, TOSHIYOSHI FUJIWARA ${ }^{4}$ and TETSUYA NAKATSURA ${ }^{1}$

\footnotetext{
${ }^{1}$ Section for Cancer Immunotherapy, Investigative Treatment Division, Research Center for Innovative Oncology,

${ }^{2}$ Division of Hepatobiliary and Pancreatic Oncology, ${ }^{3}$ Division of Digestive Surgery, National Cancer Center Hospital East, 6-5-1 Kashiwanoha, Kashiwa, Chiba 277-8577; ${ }^{4}$ Department of Gastroenterological

Surgery, Okayama University Graduate School of Medicine, Dentistry, and Pharmaceutical Sciences, 2-5-1 Shikata-cho, Okayama 700-8558; ${ }^{5}$ Department of Gastroenterology and Hepatology, Graduate School of Medical Sciences, Kumamoto University, 1-1-1 Honjo, Kumamoto 860-8556, Japan
}

Received July 9, 2011; Accepted August 25, 2011

DOI: 10.3892/ijo.2011.1202

\begin{abstract}
Glypican-3 (GPC3), a carcinoembryonic antigen, is an ideal target for anticancer immunotherapy against hepatocellular carcinoma (HCC). In this study, we attempted to compare the induction of the GPC3-specific T-cell-mediated immune response after locoregional therapies in HCC patients and tumor-bearing mice. Twenty-seven HCC patients treated with locoregional therapies, including radiofrequency ablation (RFA), surgical resection and transcatheter arterial chemoembolization (TACE), were prospectively enrolled in this study. Additionally, we performed RFA experiments using a mouse
\end{abstract}

Correspondence to: Dr Tetsuya Nakatsura, Section for Cancer Immunotherapy, Investigative Treatment Division, Research Center for Innovative Oncology, National Cancer Center Hospital East, 6-5-1 Kashiwanoha, Kashiwa, Chiba 277-8577, Japan

E-mail: tnakatsu@east.ncc.go.jp

${ }^{*}$ Contributed equally

Abbreviations: GPC3, glypican-3; HCC, hepatocellular carcinoma; RFA, radiofrequency ablation; TACE, transcatheter arterial chemoembolization; CTL, cytotoxic T lymphocyte; CT, computed tomography; TNM, tumor-node-metastasis; UICC, the Union for International Cancer Control; PBMC, peripheral blood mononuclear cell; IFN, interferon; ELISPOT, enzyme-linked immunospot; HSP105, heat shock protein 105; CMV, cytomegalovirus; AFP, $\alpha$-fetoprotein; PIVKA-II, protein induced by vitamin $\mathrm{K}$ absence or antagonist II; hTERT, human telomerase reverse transcriptase; MRP3, multidrug resistance-associated protein 3

Key words: hepatocellular carcinoma, radiofrequency ablation, glypican-3, cytotoxic T lymphocyte, immunotherapy model. GPC3-specific T-cell response was investigated pretreatment and post-treatment by an interferon- $\gamma$ enzyme-linked immunospot assay using peripheral blood mononuclear cells from HCC patients and lymph node cells from tumor-bearing mice. Circulating GPC3-specific cytotoxic T lymphocytes (CTLs) were increased in 5 of 9 patients after RFA and in 4 of 9 patients after TACE, but in only 1 of 9 patients after surgical resection. All 7 patients with GPC3-expressing HCCs exhibited an increase in GPC3-specific CTLs after RFA or TACE, whereas none of the 7 patients did after surgical resection. The number of increased GPC3-specific CTLs after RFA was significantly larger than that after surgical resection $(\mathrm{P}=0.023)$. Similarly, the frequency of GPC3-specific CTLs after RFA was significantly greater than that after surgical resection in the mouse model $(\mathrm{P}=0.049)$. We validated for the first time the stronger effect on the immune system brought by RFA compared with surgical resection for HCC patients and tumor-bearing mice. Combined treatment of RFA and immunotherapy is a reasonable strategy against HCC.

\section{Introduction}

Hepatocellular carcinoma (HCC) is one of the most common and most serious cancers worldwide (1). Locoregional therapies, including radiofrequency ablation (RFA), surgical resection, and transcatheter arterial chemoembolization (TACE), are recognized as the gold-standard therapies for HCC patients whose cancer lesions are limited to the liver (2). However, the recurrence rate remains quite high despite potentially curative treatment $(3,4)$. The reasons for this are as follows: first, a multicentric new tumor frequently occurs from underlying active hepatitis or cirrhosis and, second, a small tumor undetectable by imaging modalities frequently exists before treatment and would be left untreated (5). Therefore, the establishment of effective adjuvant therapy to prevent recurrence is urgently required, and 
clinical trials are ongoing throughout the world (6). However, at the present time, there is no universal consensus $(2,7,8)$.

Previous studies have reported that local tumor ablation treatments, such as RFA and cryoablation, not only destroy tumor tissue but also induce a marked inflammatory response both locally and systemically $(9,10)$. Unlike surgical resection, tumor ablation treatment generates tumor cell necrosis (11), followed by the release of tumor-associated antigens (12). These antigens can be uptaken, processed, and presented by dendritic cells $(10,13)$, and then an antigen-specific T-cellmediated immune response can be induced (9). If this induction is sufficiently steady and reliable, it may provide the basis for adjuvant immunotherapy, which is an attractive strategy.

Glypican-3 (GPC3) belongs to the glypican family of heparan sulfate proteoglycans that are linked to the outer surface of the cell membrane through a glycosylphosphatidylinositol anchor (14). GPC3 is one of the carcinoembryonic antigens overexpressed in HCC (15-17). We have shown that GPC3 is an ideal target for anticancer immunotherapy because its expression is specifically detected in $~ 80 \%$ of HCCs even in the early stages and is correlated with a poor prognosis (18-21). Moreover, GPC3-specific cytotoxic T lymphocytes (CTLs) have a high level of killing activity against HCC tumor cells (22). We have finished the phase I clinical trial of a GPC3derived peptide vaccine for patients with advanced HCC (unpublished data), and just started the phase II clinical trial for adjuvant therapy after curative resection or RFA.

In this study, our aim was to determine if the GPC3specific T-cell-mediated immune response is strengthened after locoregional therapies in HCC patients and tumorbearing mice. Moreover, we evaluated the hypothesis that the post-treatment immune response may provide the basis for adjuvant immunotherapy.

\section{Materials and methods}

Patient population and treatment of HCC. Twenty-seven patients with primary $\mathrm{HCC}$ were prospectively enrolled in this study from January to November 2007 at the National Cancer Center Hospital East, in Japan. The eligibility criteria included primary $\mathrm{HCC}$, which would undergo locoregional therapies with curative intent. Three treatment groups of nine patients each would undergo RFA, surgical resection, or TACE, respectively. Treatment selection in each patient was in accordance with the Japanese HCC treatment guidelines (2). Other inclusion criteria included HLA-A24 or HLA-A2 gene-positive status, as determined by commercially-available genomic DNA typing tests (Mitsubishi Chemical Medience, Tokyo, Japan), and no other active malignancy. HCC was diagnosed using dynamic computed tomography (CT). Tumor stage was assigned according to the tumor-node-metastasis (TNM) classification of the Union for International Cancer Control (UICC) (23). All RFA procedures were performed percutaneously under ultrasound guidance. Curative treatment was defined as complete necrosis of the tumor lesion confirmed by dynamic CT after RFA, a negative surgical margin confirmed histopathologically after resection, and complete lipiodol deposition after TACE.

All patients gave written informed consent before entering the study and this study was approved by the Ethics Committee of the National Cancer Center, conforming to the ethical guidelines of the 1975 Declaration of Helsinki.

Collection of blood samples and preparation of peripheral blood mononuclear cells. Venous blood (20-30 ml) from each patient was collected both before treatment and one month after treatment. Peripheral blood mononuclear cells (PBMCs) were separated from whole blood using LeucoSep ${ }^{\circledR}$ tubes (Greiner Bio-One, Frickenhausen, Germany) by means of density gradient centrifugation.

Identification of GPC3-specific CTLs in HCC patients. In order to identify GPC3-specific CTLs, the proportion of cells producing interferon (IFN)- $\gamma$ upon stimulation with GPC3 peptide was assessed by an ex vivo IFN- $\gamma$ enzyme-linked immunospot (ELISPOT) assay using pooled PBMCs from $\mathrm{HCC}$ patients. Defrosted PBMCs $\left(1 \times 10^{6}\right.$ cells/well $)$ were cultured in duplicate using 96-well flat-bottomed plates (BD Biosciences, San Jose, CA) with HLA-A24-restricted GPC3 ${ }_{298-306}$ peptide (EYILSLEEL) or HLA-A2-restricted GPC $3_{144-152}$ peptide (FVGEFFTDV) $(10 \mu \mathrm{mol} / \mathrm{l})$ with $100 \mathrm{U} / \mathrm{ml}$ recombinant human interleukin-2 (IL-2) for $20 \mathrm{~h}$. The negative control consisted of medium alone or HLA-A24- or HLA-A2-restricted heat shock protein 105 (HSP105) peptide, and the positive control included the HLA-A24- or HLA-A2-restricted cytomegalovirus (CMV) peptide. The number of spots, which indicated the presence of IFN- $\gamma$ secreting cells, was automatically counted using the Eliphoto system (Minerva Tech, Tokyo, Japan). For an exact comparison of the frequency of GPC3-specific CTLs existing at pre- and post-treatment, the obtained mean values of the number of spots with non-peptide-pulsed samples $\left(1 \times 10^{6}\right.$ PBMCs) at pre- and post-treatment were equalized and set to zero, and then the actual number of GPC3-, CMV-, or HSP105specific spots was calculated. The $\Delta$ spot was defined as the difference in the number of spots with each antigen between pre- and post-treatment.

Mice. Female BALB/c mice $\left(\mathrm{H}-2^{\mathrm{d}}\right), 6-8$ weeks of age, were obtained from Charles River Laboratories Japan (Yokohama, Japan). The mice were maintained under specific-pathogenfree conditions. All animal procedures were performed in compliance with the guidelines by the Animal Research Committee of the National Cancer Center, Japan.

Tumor cell lines. A subline of the BALB/c-derived GPC3negative colorectal adenocarcinoma cell line, Colon 26 (24), was provided by Dr Kyoichi Shimomura (Astellas Pharma, Tokyo, Japan). Colon 26/GPC3 is an established stable GPC3expressing cell line (18). The cells were cultured in RPMI-1640 medium supplemented with $10 \%$ fetal bovine serum, $100 \mathrm{U} / \mathrm{ml}$ penicillin, and $100 \mu \mathrm{g} / \mathrm{ml}$ streptomycin in humidified $5 \% \mathrm{CO}_{2}$ at $37^{\circ} \mathrm{C}$.

RFA experiment using a mouse model. The mice were shaved at the tumor area and the contralateral flank. After attachment of the electricity-conducting pad (ground pad) onto the contralateral side, an RFA needle with 5-mm active tip (Cool-tip ${ }^{\mathrm{TM}}$, Valleylab, Boulder, CO) was inserted into the middle of the tumor. Impedance could be evaluated on the RFA lesion generator system (RFG-3B model, Radionics, Burlington, MA). 
Treatment was started by delivering RFA energy. During two treatment cycles of $10 \mathrm{sec}$, the temperature could be monitored using the thermistor and thermocouple in the tip of the probe. Treatment was considered successful if a tip temperature of $60-70^{\circ} \mathrm{C}$ was reached.

Identification of GPC3-specific CTLs in mice. BALB/c mice were immunized beforehand by peptide vaccination with $\mathrm{K}^{\mathrm{d}}$-restricted GPC $3_{298-306}$ peptide (50 $\mu \mathrm{g} / \mathrm{mouse}$ ) emulsified with incomplete Freund's adjuvant twice at a 7-day interval as described previously (20). The day after the second vaccination, the mice were challenged subcutaneously with Colon 26/ GPC 3 tumor cells $\left(1 \times 10^{5}\right.$ cells/100 $\left.\mu \mathrm{l}\right)$ on their shaved back and, 5 days later, the mice underwent therapeutic RFA or surgical resection for the established tumor. After the next 5 days, the mice were sacrificed and bilateral inguinal lymph nodes were obtained. $\mathrm{CD}^{+} \mathrm{T}$ cells were isolated from lymph node cells using anti-mouse CD8 $\alpha$ (Ly-2) microbeads (Miltenyi Biotec, Bergisch Gladbach, Germany) and an IFN- $\gamma$ ELISPOT assay was performed without prior in vitro stimulation. For the IFN- $\gamma$ ELISPOT assay, CD8 ${ }^{+}$lymph node cells $\left(3 \times 10^{5}\right.$ cells/ well) were used as effector cells, and Colon 26 and Colon 26/ GPC 3 cells $\left(3 \times 10^{4}\right.$ cells/well) as target cells. These cells were cultured in duplicate using 96-well flat-bottomed plates (BD Biosciences) with $100 \mathrm{U} / \mathrm{ml}$ recombinant murine IL-2 for $20 \mathrm{~h}$. The number of spots after RFA or surgical resection was compared with that without treatment.

Immunohistochemical analysis. To investigate GPC3 expression in HCC tissues, we performed immunohistochemical staining of GPC3 in biopsy specimens or resected specimens from HCC patients. The paraffin-embedded blocks were analyzed using monoclonal anti-GPC3 antibody (dilution 1:300, BioMosaics, Burlington, VT) as described previously $(17,21)$. The results were classified into two groups according to the area of GPC3positive staining cells as follows: -, negative $(<10 \%)$ and + , positive $(\geq 10 \%)$.

To investigate tumor-infiltrating lymphocytes, we performed immunohistochemical staining of CD4 and CD8 in resected specimens from an HCC patient using monoclonal anti-CD4 or CD8 antibody (dilution 1:20, Novocastra, Newcastle upon Tyne, UK).

Statistical analysis. Statistical analyses were performed using $\chi^{2}$ test, Mann-Whitney U test, or Kruskal-Wallis rank test. Differences were considered significant at $\mathrm{P}<0.05$. Data were analyzed with the StatView 5.0 software package (Abacus Concepts, Calabasas, CA).

\section{Results}

Demographics and clinical characteristics. The characteristics of all 27 patients are represented in Table I. The three groups of 9 patients received RFA (RFA1-9), surgical resection (RES1-9), or TACE (TAE1-9), respectively. Among them, 21 patients had the HLA-A24 gene and 7 had the HLA-A2 gene. One patient had both HLA-A24 and -A2, and the HLA-A2-restricted GPC $3_{144-152}$ peptide was used for the IFN- $\gamma$ ELISPOT assay in this patient. Among the three treatment groups, tumor size in the RFA group (mean: $16.4 \mathrm{~mm}$ ) was significantly smaller than that in the resection group (mean: $43.2 \mathrm{~mm})(\mathrm{P}=0.001)$ and the TACE group (mean: $44.1 \mathrm{~mm})(\mathrm{P}=0.001)$. Similarly, tumor stage in the RFA group was less advanced than that in the resection group $(\mathrm{P}=0.018)$ and TACE group $(\mathrm{P}=0.005)$. There was no statistically significant difference in Child-Pugh classification grade among the three groups $(\mathrm{P}=0.128)$. In this study, all treatments were considered to be curative according to the definitions described in Materials and methods. Moreover, all groups reduced the levels of $\alpha$-fetoprotein (AFP) and protein induced by vitamin $\mathrm{K}$ absence or antagonist II (PIVKA-II) in most of HCC patients after treatment (data not shown). The diagnosis of HCC was histopathologically confirmed by biopsy specimens or resected specimens from 21 patients. GPC3 expression was detected by immunohistochemical staining in 14 of 21 patients.

Analysis of GPC3-specific CTLs in HCC patients. As shown in Table I, GPC3-specific CTLs were detected in 11 and 15 of 27 patients at pre- and post-treatment, respectively. In total, 19 patients had GPC3-specific CTLs at either pre- or posttreatment. There was no statistically significant correlation between the presence of GPC3-specific CTLs and clinical features, including HLA-A type $(\mathrm{P}=0.126)$, age $(\mathrm{P}=0.750)$, gender $(\mathrm{P}=0.764)$, HCV infection $(\mathrm{P}=0.674)$, HBV infection $(\mathrm{P}=0.764)$, Child-Pugh classification grade $(\mathrm{P}=0.404)$, tumor multiplicity $(\mathrm{P}=0.674)$, tumor size $(\mathrm{P}=0.650), \mathrm{HCC}$ staging $(\mathrm{P}=0.155)$, serum AFP level $(\mathrm{P}=0.288)$, and serum PIVKA-II level $(\mathrm{P}=0.094)$. Among the 21 patients who had the information about GPC3 expression in their HCC tissue, patients with GPC3-expressing HCCs had GPC3-specific CTLs more frequently than those with GPC3-negative HCCs, but the difference was not statistically significant $(\mathrm{P}=0.053)$.

Changes in GPC3-specific CTLs between before and after treatment. In order to analyze the effect of anticancer treatment on GPC3-specific T-cell response, we compared the frequency of GPC3-specific CTLs in PBMCs before treatment with that after treatment. As shown in Table I and Fig. 1, an increase in GPC3-specific CTLs was found in 5 of 9 patients after RFA and in 4 of 9 after TACE, but in only 1 of 9 patients after resection. Of note, all of the 7 patients with GPC3-expressing HCCs exhibited an increase in GPC3-specific CTLs after RFA or TACE, whereas none of the 7 patients with GPC3expressing HCCs did after surgical resection. The $\Delta$ spot of GPC3 in the RFA group (mean: 24.4 spots) was larger than that in the resection group (mean: -7.2 spots) $(\mathrm{P}=0.023)$. The $\triangle$ spot of GPC3 in the TACE group (mean, 36.9 spots) was also larger than that in the resection group, but the difference was not statistically significant $(\mathrm{P}=0.096)$. In contrast, the $\Delta$ spot of CMV showed no difference among the three groups $(\mathrm{P}=0.498)$. Neither the existence of GPC3-specific CTLs before or after treatment, nor the changes between before and after treatment had statistically significant correlation with patient survival according to the log-rank test in each treatment group (neither disease-free nor overall), with the 27-month mean follow-up period (data not shown).

The representative data on changes in CT images and serum levels of tumor markers between before and after treatment is shown in Fig. 2. All three patients (RFA3, RES6, and TAE5) had GPC3-expressing HCCs. Both the CT images and 


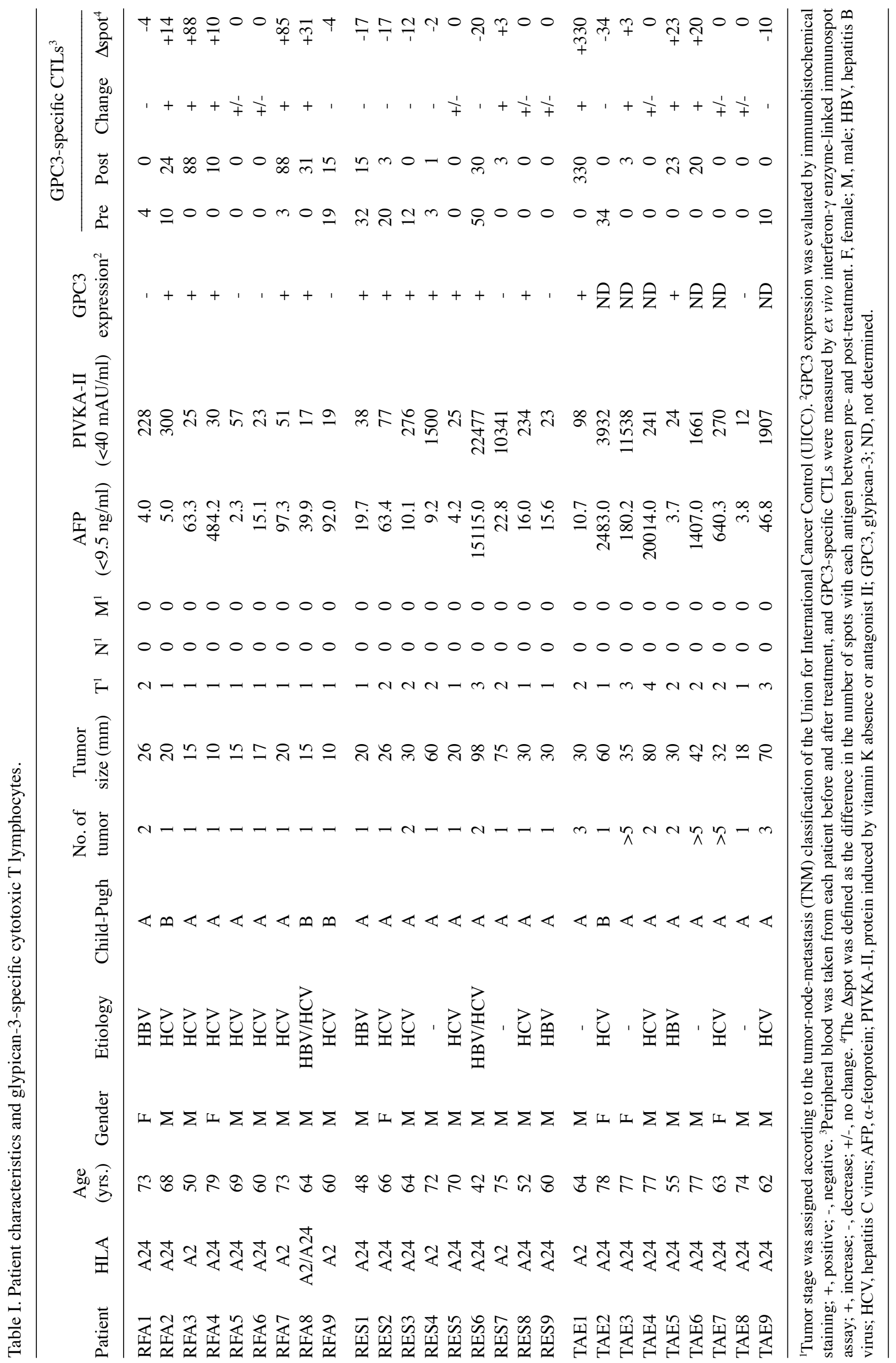




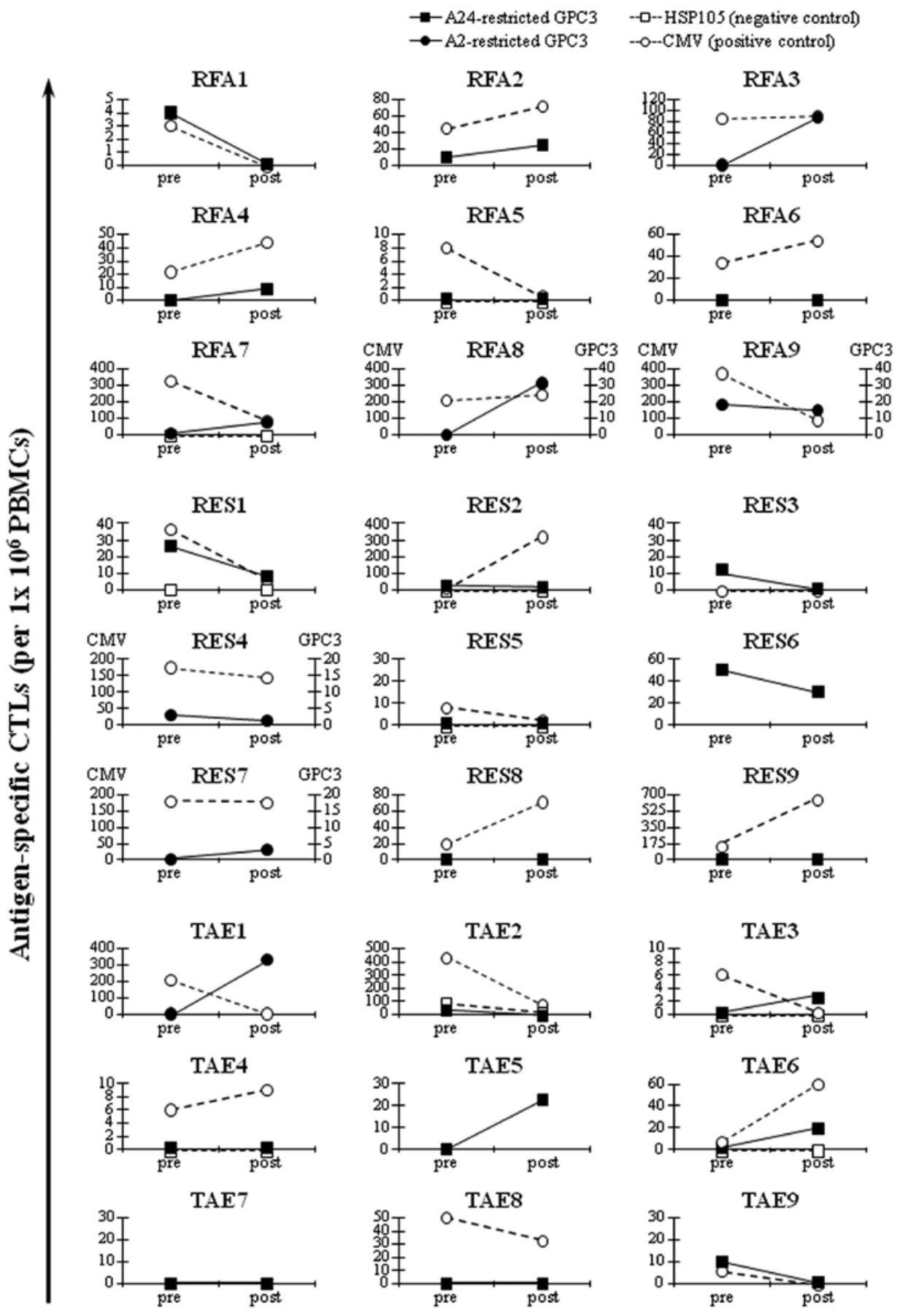

Figure 1. Kinetics of glypican-3 (GPC3)-specific CTLs between before and after treatment in each patient. A direct ex vivo interferon- $\gamma$ enzyme-linked immunospot assay of PBMCs was performed before treatment and one month after treatment. The data are expressed as the number of interferon- $\gamma$ producing cells, which indicate the CTLs specific with HLA-A24-restricted GPC3 $3_{298-306}$ peptide (EYILSLEEL) (-) or HLA-A2-restricted GPC $3_{144-152}$ peptide (FVGEFFTDV) (•). Heat shock protein 105 (HSP105) peptide ( $\square$ ) and cytomegalovirus (CMV) peptide (o) were used as the negative and positive control, respectively.

kinetics of tumor markers indicated that their treatment was effective. The frequency of GPC3-specific CTLs increased after RFA (RFA3) and TACE (TAE5), whereas it decreased after surgical resection (RES6).

RFA has the potential to strongly induce T-cell-mediated immune response: A case report. A 70-year-old woman was admitted because of recurrent HCCs. Thirteen months earlier, the patient had undergone RFA for primary HCC located in the $\mathrm{S} 5 / 8$ region of the liver. CT detected two recurrent HCCs: one was contiguous to the previously ablated $\mathrm{S} 5 / 8$ region and the other was a distant tumor located in the S6 region. We performed surgical resection for these recurrent HCCs. Immunohistochemical examination of CD8 in the resected tumors revealed that a marked number of $\mathrm{CD}^{+} \mathrm{T}$ cells had infiltrated not only into the surrounding recurrent tumor but also into the distant recurrent tumor after RFA (Fig. 3). On the other hand, few $\mathrm{CD}^{+} \mathrm{T}$ cells were observed in these tumors (data not shown). Immunohistochemical analyses showed the expression of GPC3 and HLA class I in these tumors (data not 


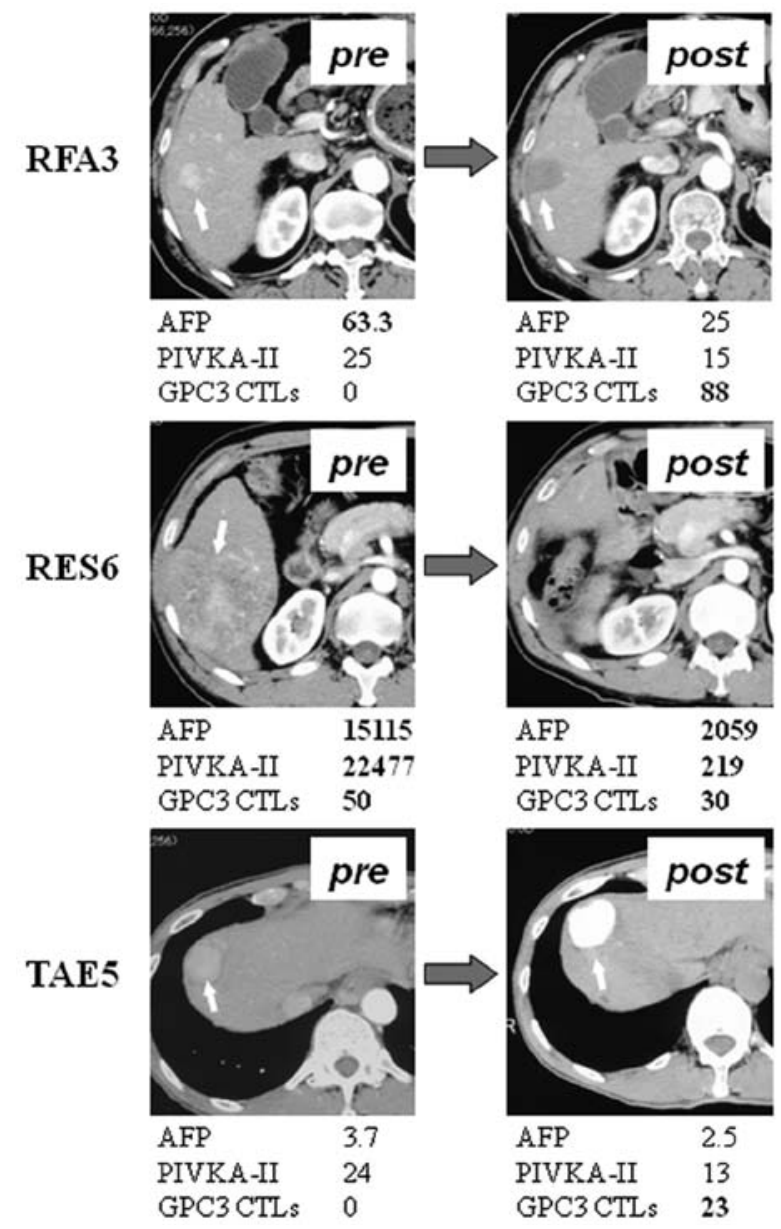

Figure 2. Changes in computed tomography images, serum levels of tumor markers, including $\alpha$-fetoprotein (AFP) and protein induced by vitamin $\mathrm{K}$ absence or antagonist II (PIVKA-II), and glypican-3 (GPC3)-specific CTLs in PBMCs between before and after treatment in patients RFA3, RES6, and TAE5. White arrows indicate nodules of hepatocellular carcinoma at pre- and post-treatment. The bold letters show the abnormal levels of tumor markers or the positive response of GPC3 specific CTLs.

shown). These findings suggest that RFA not only activates the immune response systemically but also induces local infiltration of CTLs into the tumors.

Analysis of immune response induced by RFA in a mouse model. The experimental schedule is shown in Fig. 4A. The IFN $-\gamma$ ELISPOT assay with CD8 ${ }^{+} \mathrm{T}$ cells from the lymph nodes of mice demonstrated that the number of spots against both Colon $26(\mathrm{P}=0.049)$ and Colon 26/GPC3 $(\mathrm{P}=0.049)$ was larger after RFA compared to without treatment. On the other hand, the number of spots did not increase after surgical resection. These results suggest that RFA induced a significantly larger number of both Colon 26- and Colon 26/GPC3-reactive CTLs compared to no treatment or surgical resection (Fig. 4B).

The difference in number of spots between Colon 26 and Colon 26/GPC3 in each mouse, which represents GPC3specific CTLs, is shown in Fig. 4C. As an effect of prior peptide vaccination, GPC3-specific CTLs were detected in the no treatment group. The frequency of GPC3-specific CTLs increased after RFA and decreased after surgical resection. As a result, the frequency of GPC3-specific CTLs after RFA was significantly greater than that after surgical resection $(\mathrm{P}=0.049)$.
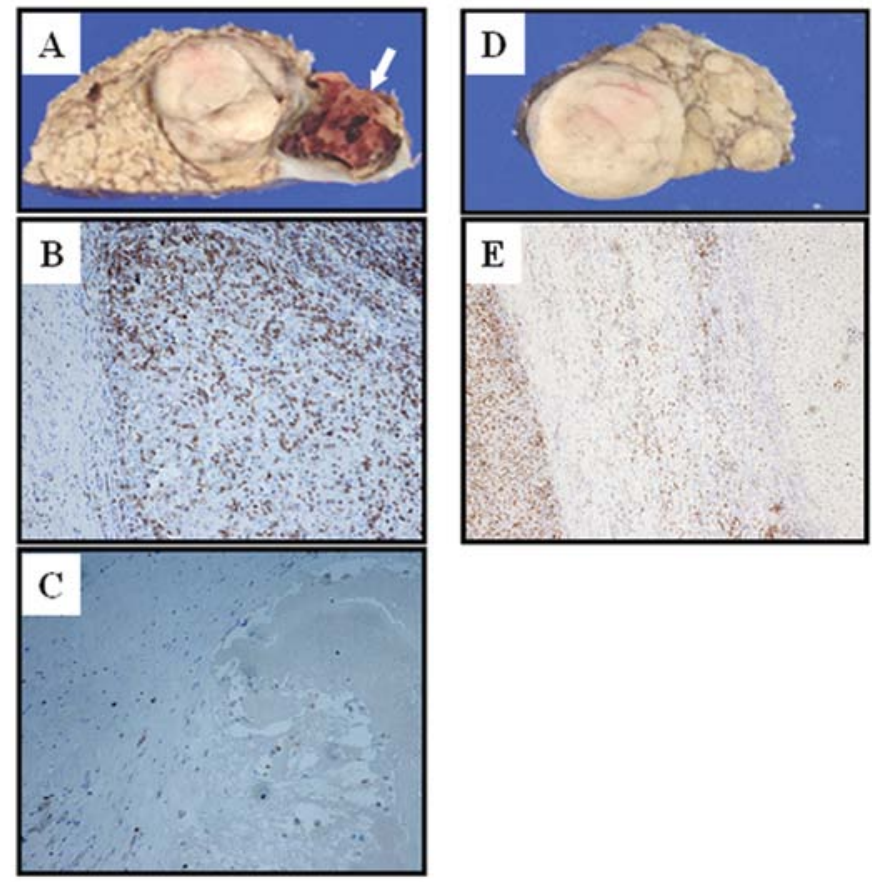

Figure 3. Macroscopic features and immunohistochemical examination of $\mathrm{CD}^{+} \mathrm{T}$ cells in the resected tumors that had recurred after radiofrequency ablation. (A and D) show the cut surface of the resected specimens. (A) The white arrow indicates the post-ablated lesion to which a recurrent tumor was contiguous. The other recurrent tumor was distant from the post-ablated lesion (D). A marked number of $\mathrm{CD}^{+} \mathrm{T}$ cells had infiltrated into the contiguous recurrent tumor (B) and the distant recurrent tumor (E), whereas few $\mathrm{CD}^{+} \mathrm{T}$ cells had infiltrated into the post-ablated necrotic lesion $(\mathrm{C})$. Magnification x100 (B and C) and x40 (E).

These results suggest that RFA induced a significantly larger number of GPC3-specific CTLs compared to surgical resection (Fig. 4C).

\section{Discussion}

We previously reported that $39 \%$ of HCC patients had detectable GPC3-specific CTLs by a direct ex vivo IFN- $\gamma$ ELISPOT assay (25). In this study, GPC3-specific CTLs were detectable before treatment in 11 of 27 patients (41\%). Additionally, when we analyzed the patients with a prior treatment for HCCs using the same methods, 11 of $21(52 \%)$ patients had detectable GPC3-specific CTLs (data not shown). These results are favorable for anticancer immunotherapy because the antigenspecific T-cell-mediated immune response could be detected without in vitro stimulation. As for frequency, GPC3-specific CTLs were detectable in $\sim 40 \%$ of HCC patients, whereas AFP-, human telomerase reverse transcriptase (hTERT)-, and multidrug resistance-associated protein 3 (MRP3)-specific CTLs have been detected in 5-20, 6-12, and 14-21\% of HCC patients with a single epitope peptide, respectively (26-28). As for tumor stages, a GPC3-specific immune response is frequently detected even in the early stages (24), whereas AFP-specific CTLs are more frequently detected in patients with advanced HCC (26). These results suggest that GPC3 has strong immunogenicity and GPC3-specific T-cell-mediated immunotherapy is suitable for adjuvant therapy against HCC because the induction of tumor-specific immune response in 
A

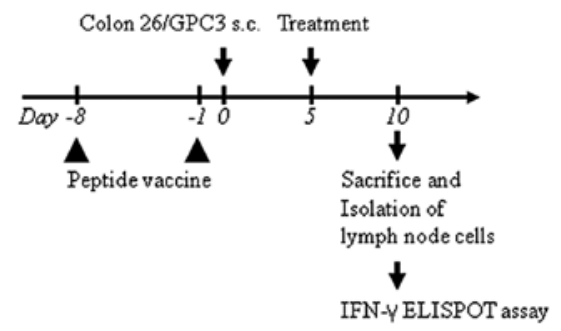

B

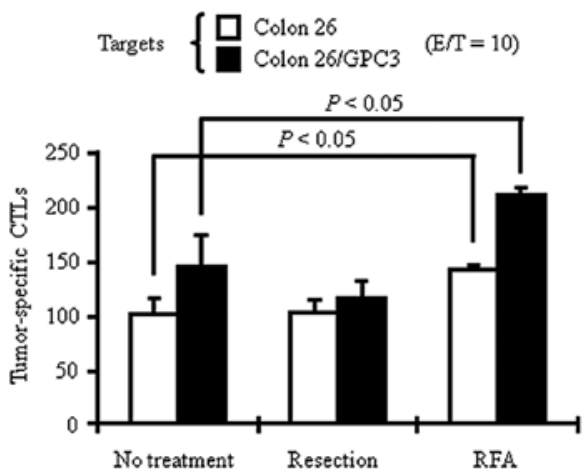

$\mathrm{C}$

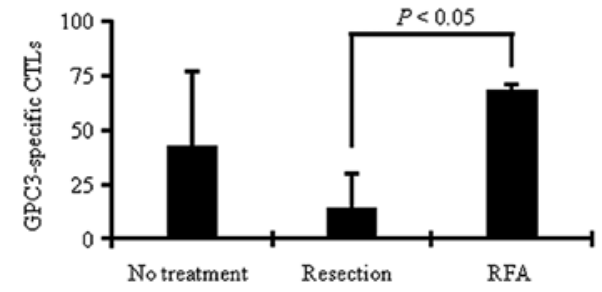

Figure 4. Investigation of the glypican-3 (GPC3)-specific immune response in a mouse model. (A) Experiment schedule. (B) An ex vivo interferon (IFN)- $\gamma$ enzyme-linked immunospot (ELISPOT) assay of CD8 ${ }^{+} 1 y m p h$ node cells (effector, $3 \times 10^{5}$ cells/well) against Colon 26 and Colon 26/GPC3 (target, $3 \times 10^{4}$ cells/well). No treatment column indicates the group of mice that received only the peptide vaccination and no therapy for the established tumor. The data are expressed as the mean + SD. Three mice were used for each group. Effector/target ratio=10. (C) The frequency of GPC3-specific CTLs, which is calculated from the difference in the number of spots between Colon 26 and Colon 26/GPC3 in each mouse.

the early stages would be more effective for suppression of tumor growth.

The association between the induction of an antigenspecific immune response and the antigen expression in tumor tissue remains unclear. In this study, we obtained the result that the presence of GPC3-specific CTLs in PBMCs potentially had a positive correlation with GPC3 expression in tumor tissue, but the correlation was not statistically significant. On the other hand, Mizukoshi et al showed a negative correlation between the frequency of MRP3-specific CTLs and MRP3 expression level (28). Moreover, Benavides et al showed that even antigennaïve patients had pre-existing immunity (29). First, this may be because of tumor heterogeneity of cancer tissue. In most cases, the whole tumor cannot be evaluated and, in the case of truly antigen-naïve patients, antigen-specific CTLs cannot exist in theory. Second, antigen expression may be negative if antigen-specific CTLs have killed all of the antigen-expressing tumor cells as described by Jäger et al (30). As for the changes in an antigen-specific immune response between before and after treatment, in this study, we showed impressive data that all patients with GPC3-expressing HCCs exhibited an increase in GPC3-specific CTLs after RFA or TACE, whereas no patient with GPC3-expressing HCCs did after surgical resection.

This is the first study to compare locoregional therapies, including RFA, surgical resection, and TACE, in terms of antigen-specific T-cell response in HCC patients and tumorbearing mice. Half the patients after RFA or TACE showed an increase in GPC3-specific CTLs, which might have been induced by the treatment, whereas only 1 of 9 patients after resection showed an increase and more than half the patients after resection showed a decrease. Similarly, the frequency of GPC3-specific CTLs increased after RFA and decreased after resection in a mouse model. These results suggest that RFA induced a stronger GPC3-specific immune response compared to surgical resection. RFA destroys tumor tissue and causes local necrosis followed by the release of tumor-associated antigens (12), whereas all of the tumor-associated antigens must be completely removed after resection. With regard to TACE, whereas the results of an IFN- $\gamma$ ELISPOT assay after TACE were as encouraging as that after RFA, we have no other favorable data on the immune response after TACE. Although further investigation is required, TACE, which is also a necrosis-inducing treatment, might induce an antigen-specific immune response.

A limitation of this study is the patient selection in the three kinds of locoregional therapy. Current treatment guidelines for HCC including the Japanese ones, which we followed in this study, recommend RFA to earlier HCCs and TACE to more advanced HCCs than those which receive surgical resection (2,31-33). Therefore, selection bias is unavoidable under the circumstances. To overcome this problem, we added a murine study. The advantage of RFA over surgical resection in the induction of GPC3-specific CTLs was demonstrated also in a mouse model.

The correlation between antitumor immune response and clinical response is controversial. In this study, a significant contribution of GPC3-specific CTLs toward an optimal prognosis was not demonstrated. Mizukoshi et al reported that enhancement of T-cell response did not last for long and did not contribute to the prevention of HCC recurrence (34). In view of the highly complex nature of the human immune system, patient prognoses might not be determined only by the CTL response. Previous studies have demonstrated that the release of tumor-derived antigens by necrosis-inducing treatment causes sufficient signaling to activate not only antigen-specific CTL response but also antigen-specific helper T-cell response $(35,36)$, antigen-specific antibody response (36), and nonantigen-specific natural killer cell response (37). However, the mechanisms for cancer escape from immunosurveillance would suppress the efficiency of these immune responses (38). In the literature, tumor-infiltrating lymphocytes in HCC are associated with better prognosis (39), but, in our case, tumor-infiltrating CTLs were actually insufficient for suppression of cancer recurrence despite the massive infiltration. For successful anticancer immunotherapy, the development of an innovative strategy to link antitumor immune response with clinical response and to provide a survival benefit for cancer patients is necessary, and so we have just started the clinical trial of a GPC3-derived peptide vaccine for adjuvant therapy after RFA. 
In conclusion, our results demonstrate that RFA has a stronger effect on the immune system compared with surgical resection. Although further investigation is necessary, the data on immune response support the rationale for combined immunotherapy for HCC patients.

\section{Acknowledgements}

D.N. would like to thank the Foundation for Promotion of Cancer Research (Japan) for the Third-Term Comprehensive Control Research for Cancer for awarding them a research resident fellowship. This study was supported in part by Health and Labor Science Research Grants for Research on Hepatitis from the Ministry of Health, Labor, and Welfare, Japan. J.F. received honoraria and consulting fee from Bayer, Chugai, Eisai, Eli Lilly, Taiho and Phizer; J.F.'s institute received grants from Pfizer and Bayer.

\section{References}

1. Parkin DM: Global cancer statistics in the year 2000. Lancet Oncol 2: 533-543, 2001

2. Makuuchi M, Kokudo N, Arii S, et al: Development of evidencebased clinical guidelines for the diagnosis and treatment of hepatocellular carcinoma in Japan. Hepatol Res 38: 37-51, 2008.

3. Imamura H, Matsuyama Y, Tanaka E, et al: Risk factors contributing to early and late phase intrahepatic recurrence of hepatocellular carcinoma after hepatectomy. J Hepatol 38: 200-207, 2003.

4. Tateishi R, Shiina S, Yoshida H, et al: Prediction of recurrence of hepatocellular carcinoma after curative ablation using three tumor markers. Hepatology 44: 1518-1527, 2006.

5. Nobuoka D, Kato Y, Gotohda N, et al: Postoperative serum alpha-fetoprotein level is a useful predictor of recurrence after hepatectomy for hepatocellular carcinoma. Oncol Rep 24: 521-528, 2010.

6. Printz C: Clinical trials of note. Sorafenib as adjuvant treatment in the prevention of disease recurrence in patients with hepatocellular carcinoma (HCC) (STORM). Cancer 115: 4646, 2009.

7. Schwartz JD, Schwartz M, Mandeli J and Sung M: Neoadjuvant and adjuvant therapy for resectable hepatocellular carcinoma: review of the randomised clinical trials. Lancet Oncol 3: 593-603, 2002.

8. Ishii $\mathrm{H}$, Yamamoto $\mathrm{J}$ and Ikari T: Adjuvant treatments for resectable hepatocellular carcinoma. J Hepatobiliary Pancreat Surg 15: 459-462, 2008.

9. Wissniowski TT, Hänsler J, Neureiter D, et al: Activation of tumor-specific $\mathrm{T}$ lymphocytes by radio-frequency ablation of the VX2 hepatoma in rabbits. Cancer Res 63: 6496-6500, 2003.

10. den Brok MH, Sutmuller RP, Nierkens S, et al: Efficient loading of dendritic cells following cryo and radiofrequency ablation in combination with immune modulation induces anti-tumour immunity. Br J Cancer 95: 896-905, 2006.

11. McGahan JP, Brock JM, Tesluk H, Gu WZ, Schneider P and Browning PD: Hepatic ablation with use of radio-frequency electrocautery in the animal model. J Vasc Interv Radiol 3: 291-297, 1992.

12. Yang WL, Nair DG, Makizumi R, et al: Heat shock protein 70 is induced in mouse human colon tumor xenografts after sublethal radiofrequency ablation. Ann Surg Oncol 11: 399-406, 2004.

13. Ali MY, Grimm CF, Ritter M, et al: Activation of dendritic cells by local ablation of hepatocellular carcinoma. J Hepatol 43 : 817-822, 2005.

14. Filmus J: The contribution of in vivo manipulation of gene expression to the understanding of the function of glypicans. Glycoconj J 19: 319-323, 2002.

15. Nakatsura T, Yoshitake Y, Senju S, et al: Glypican-3, overexpressed specifically in human hepatocellular carcinoma, is a novel tumor marker. Biochem Biophys Res Commun 306: 16-25, 2003.

16. Nakatsura $T$ and Nishimura $Y$ : Usefulness of the novel oncofetal antigen glypican-3 for diagnosis of hepatocellular carcinoma and melanoma. BioDrugs 19: 71-77, 2005.
17. Shirakawa H, Kuronuma T, Nishimura Y, et al: Glypican-3 is a useful diagnostic marker for a component of hepatocellular carcinoma in human liver cancer. Int J Oncol 34: 649-656, 2009.

18. Nakatsura T, Komori H, Kubo T, et al: Mouse homologue of a novel human oncofetal antigen, glypican-3, evokes T-cellmediated tumor rejection without autoimmune reactions in mice. Clin Cancer Res 10: 8630-8640, 2004.

19. Komori H, Nakatsura T, Senju S, et al: Identification of HLA-A2or HLA-A24-restricted CTL epitopes possibly useful for glypican-3-specific immunotherapy of hepatocellular carcinoma. Clin Cancer Res 12: 2689-2697, 2006.

20. Motomura Y, Ikuta Y, Kuronuma T, et al: HLA-A2 and -A24-restricted glypican-3-derived peptide vaccine induces specific CTLs: preclinical study using mice. Int J Oncol 32: 985-990, 2008.

21. Shirakawa H, Suzuki H, Shimomura M, et al: Glypican-3 expression is correlated with poor prognosis in hepatocellular carcinoma. Cancer Sci 100: 1403-1407, 2009.

22. Yoshikawa T, Nakatsugawa M, Suzuki S, et al: HLA-A2restricted glypican-3 peptide-specific CTL clones induced by peptide vaccine show high avidity and antigen-specific killing activity against tumor cells. Cancer Sci 102: 918-925, 2011.

23. Sobin LH and Wittekind C: UICC: TNM Classification of Malignant Tumors. 6th edition, Wiley-Liss, New York, pp81-83, 2002 .

24. Tanaka Y, Eda H, Tanaka T, et al: Experimental cancer cachexia induced by transplantable colon 26 adenocarcinoma in mice. Cancer Res 50: 2290-2295, 1990.

25. Hayashi E, Motomura Y, Shirakawa H, et al: Detection of glypican-3-specific CTLs in chronic hepatitis and liver cirrhosis. Oncol Rep 22: 149-154, 2009.

26. Mizukoshi E, Nakamoto Y, Tsuji H, Yamashita T and Kaneko S: Identification of alpha-fetoprotein-derived peptides recognized by cytotoxic $\mathrm{T}$ lymphocytes in HLA-A $24^{+}$patients with hepatocellular carcinoma. Int J Cancer 118: 1194-1204, 2006.

27. Mizukoshi E, Nakamoto Y, Marukawa Y, et al: Cytotoxic T cell responses to human telomerase reverse transcriptase in patients with hepatocellular carcinoma. Hepatology 43: 1284-1294, 2006.

28. Mizukoshi E, Honda M, Arai K, Yamashita T, Nakamoto Y and Kaneko S: Expression of multidrug resistance-associated protein 3 and cytotoxic $\mathrm{T}$ cell responses in patients with hepatocellular carcinoma. J Hepatol 49: 946-954, 2008.

29. Benavides LC, Gates JD, Carmichael MG, et al: The impact of HER2/neu expression level on response to the E75 vaccine: from U.S. Military Cancer Institute Clinical Trials Group Study I-01 and I-02. Clin Cancer Res 15: 2895-2904, 2009.

30. Jäger E, Ringhoffer M, Karbach J, Arand M, Oesch F and Knuth A: Inverse relationship of melanocyte differentiation antigen expression in melanoma tissues and $\mathrm{CD} 8^{+}$cytotoxic-Tcell responses: evidence for immunoselection of antigen-loss variants in vivo. Int J Cancer 66: 470-476, 1996.

31. Llovet JM, Burroughs A and Bruix J: Hepatocellular carcinoma. Lancet 362: 1907-1917, 2003.

32. Bruix J and Sherman M:Practice Guidelines Committee,American Association for the Study of Liver Diseases: Management of hepatocellular carcinoma. Hepatology 42: 1208-1236, 2005.

33. Omata M,Lesmana LA, Tateishi R, et al: Asian Pacific Association for the Study of the Liver consensus recommendations on hepatocellular carcinoma. Hepatol Int 4: 439-474, 2010.

34. Mizukoshi E, Nakamoto Y, Arai K, et al: Enhancement of tumorspecific T-cell responses by transcatheter arterial embolization with dendritic cell infusion for hepatocellular carcinoma. Int J Cancer 126: 2164-2174, 2010.

35. Ayaru L, Pereira SP, Alisa A, et al: Unmasking of alphafetoprotein-specific CD4(+) T cell responses in hepatocellular carcinoma patients undergoing embolization. J Immunol 178: 1914-1922, 2007.

36. Widenmeyer M, Shebzukhov Y, Haen SP, et al: Analysis of tumor antigen-specific $\mathrm{T}$ cells and antibodies in cancer patients treated with radiofrequency ablation. Int J Cancer 128: 2653-2662, 2011.

37. Zerbini A, Pilli M, Laccabue D, et al: Radiofrequency thermal ablation for hepatocellular carcinoma stimulates autologous NK-cell response. Gastroenterology 138: 1931-1942, 2010.

38. Zerbini A, Pilli M, Penna A, et al: Radiofrequency thermal ablation of hepatocellular carcinoma liver nodules can activate and enhance tumor-specific T-cell responses. Cancer Res 66: 1139-1146, 2006.

39. Wada Y, Nakashima O, Kutami R, Yamamoto O and Kojiro M: Clinicopathological study on hepatocellular carcinoma with lymphocytic infiltration. Hepatology 27: 407-414, 1998. 